\title{
Angiografia como método de diagnóstico da síndrome do desfiladeiro torácico neurovascular. A propósito de um caso(*)
}

\author{
Angiography as a diagnostic tool in the neurovascular \\ outlet syndrome. A clinical case
}

\author{
Margarida Cruz ${ }^{(1)}$, António Alves de Matos $^{(2)}$, Tiago Saldanha ${ }^{(3)}$ e Jaime C. Branco ${ }^{(4)}$
}

\section{RESUMO}

O termo síndrome do desfiladeiro torácico (SDT) é usado para descrever o quadro clínico atribuível à compressão do plexo braquial, artéria e veia subclávias na região designada por desfiladeiro torácico. Até hoje, nenhum exame foi considerado gold standard para o diagnóstico. Os autores descrevem o caso de uma adolescente de 16 anos observada na consulta de Reumatologia por omalgia direita com 3 meses de evolução, acompanhada de disestesias do antebraço e mão homolaterais, desencadeadas por movimentos de abdução do ombro. Na observação, a manobra de hiperabdução do membro superior direito desencadeava os sintomas e um enfraquecimento significativo do pulso radial e da pressão arterial, que não sucediam no membro superior contralateral, tendo sido diagnosticada síndrome do desfiladeiro torácico à direita. Foi realizada tomografia axial computorizada (TAC) da região torácica, que não revelou massas infra-claviculares nem axilares à direita. $\mathrm{O}$ ecodoppler do membro superior mostrou uma onda trifásica na artéria subclávia, realizou-se também angiografia das artérias subclávia e axilar do membro superior direito. Esta apresentou-se normal em posição neutra, mas, com o braço em abdução máxima, observou-se uma redução do contraste no interior da artéria axilar. Os autores propõem que, quando a clínica for sugestiva de SDT e existir uma suspeita de compressão vascular, deve ser realizada angiografia das artérias subclávia e axilar, para localizar e caracterizar a compressão. A SDT dessa paciente foi atribuída a uma hipotonia muscular do ombro, que foi ultrapassada após fisioterapia com exercícios de reforço dos músculos elevadores do ombro.

Palavras-chave: Síndrome do desfiladeiro torácico, angiografia, diagnóstico.

\begin{abstract}
The term "thoracic outlet syndrome" describes a set of manifestations corresponding to the entrapment of the neurovascular bundle that comprises the brachial plexus, axillary vein and artery in a region named thoracic outlet. We still don't have a gold standard diagnostic tool, although different clinical, radiographic and electrodiagnostic tests have been described. The authors describe the case of a 16-year-old female adolescent who came to the ambulatory of the Rheumatology Department complaining of right shoulder pain and ipsilateral forearm and hand disesthesias for 3 months, which were aggravated with arm abduction movements. The right arm hyperabduction maneuver was associated with the symptoms and significant weakening of ipsilateral radial pulse and arterial pressure, but not in the contralateral arm, so a right thoracic outlet syndrome (TOS) was diagnosed. Thoracic axial computerized tomography didn't show any infraclavicular or axillary tumors. The axillary Doppler showed a triphasic wave in the subclavian artery, which lead to the performance of a right subclavian and axillary arteries angiography. It was normal in neutral position but, with arm hyperabduction, a reduction in the amount of contrast in the axillary artery was evident, meaning a local entrapment. The authors propose that, when the clinical evaluation suggests TOS and an arterial compression is suspected, an angiography should be ordered in order to localize and characterize it. The cause of this compression was considered to be a shoulder muscle hipotonia, which was successfully compensated by a set of stretching and reinforcement exercises.
\end{abstract}

Keywords: thoracic outlet syndrome, angiography, diagnosis.

\footnotetext{
* Hospital de Egas Moniz. Recebido em 9/1/2003. Aprovado, após revisão, em 7/7/2003.

1. Assistente Eventual de Reumatologia, Unidade de Reumatologia, Hospital de Egas Moniz, Lisboa, Portugal.

2. Assistente Graduado de Reumatologia, Unidade de Reumatologia, Hospital de Egas Moniz, Lisboa, Portugal.

3. Assistente Hospitalar de Radiologia, Serviço de Radiologia, Hospital de Egas Moniz, Lisboa, Portugal.

4. Chefe de Serviço de Reumatologia; Diretor da Unidade de Reumatologia, Hospital de Egas Moniz, Lisboa; Professor Agregado de Reumatologia na Faculdade de Ciências Médicas da Universidade Nova de Lisboa, Portugal.

Endereço para correspondência: Margarida Cruz. Unidade de Reumatologia, Hospital de Egas Moniz, Rua da Junqueira, 126, CEP 1349-019, Lisboa, Portugal, e-mail: margarida.cruz@iol.pt
} 


\section{INTRODUÇÃO}

\section{ANATOMIA}

O suprimento neurovascular dos membros superiores consiste no feixe constituído por: 1. Artéria subclávia, que sai do tórax sobre a primeira costela, entre as porções anterior e média do músculo escaleno. 2. Plexo braquial, formado pela junção das raízes nervosas C5 a T1 e cujo trajeto é idêntico ao da artéria, situando-se posterior e superiormente a esta. 3. Veia subclávia, que não atravessa o triângulo interescaleno, passando à frente do escaleno anterior. Estes três componentes, a meio do seu trajeto, passam no intervalo entre a primeira costela e a clavícula, denominado espaço costoclavicular ou desfiladeiro torácico. Todos entram na axila passando sob o músculo pequeno peitoral, junto à sua inserção na apófise coracóide ${ }^{(1)}$.

\section{DIAGNÓSTICO}

O termo síndrome do desfiladeiro torácico (thoracic outlet syndrome) foi introduzido por Peet et al. em $1956^{(2)}$ e é utilizado para descrever um quadro clínico atribuído à compressão do plexo braquial, artéria e veia subclávias na região designada por desfiladeiro torácico ${ }^{(1,3)}$.

O diagnóstico desta entidade baseia-se principalmente nas queixas subjetivas de dor e/ou parestesias nos membros superiores, permanecendo parcialmente controverso, pela dificuldade em quantificar estes sintomas. Até hoje, nenhum exame foi considerado gold standard para o diagnóstico, apesar de terem sido descritos vários testes clínicos, radiográficos e eletrodiagnósticos. A avaliação clínica permanece como um componente importante no diagnóstico. As parestesias e dormência são atribuídas à compressão nervosa na região do plexo braquial, e a componente dolorosa associada pode estar relacionada com alterações do balanço muscular dos músculos do pescoço, ombros e região dorsal superior ${ }^{(4)}$.

A síndrome do desfiladeiro torácico (SDT) pode ser dividida, teoricamente, em três subgrupos: 1. SDT neurológica, em que existe apenas envolvimento do plexo braquial. 2. SDT com compromisso vascular, quando este se associa ao neurológico. 3. SDT apenas vascular, afetando a artéria e/ou a veia subclávias ${ }^{(1)}$.

\section{COMPRESSÃO NERVOSA E VASCULAR}

Certas posturas do membro superior, cabeça e coluna vertebral que são assumidas no trabalho, em casa e durante o sono podem ter três conseqüências importantes: 1) Pressão direta sobre os nervos, em locais suscetíveis de compressão, provocando edema e fibrose em caso de cronicidade. 2) Posturas mantidas podem proporcionar o encurtamento de alguns músculos e, quando se estiram ou utilizam esses músculos, eles podem tornar-se dolorosos e provocar compressão nervosa. 3) Enfraquecimento de alguns músculos, com sobreutilização compensadora de outros, ou seja, alteração do equilíbrio muscular ${ }^{(3)}$. As causas mais freqüentemente relatadas de SDT são a existência de uma costela cervical ou de uma megaapófise transversa de C7, a hipertrofia dos músculos escalenos e a síndrome do escaleno anticus, com hipotonia da musculatura do ombro ${ }^{(4)}$.

O início dos sintomas de SDT geralmente é insidioso e freqüentemente relacionado com posturas durante o sono, em que os membros superiores ficam acima da cabeça e os cotovelos fletidos. Os sintomas consistem em parestesias e dormência, especialmente ao longo do bordo cubital do antebraço e mão. As atividades que exacerbam as queixas são as que colocam o membro superior em abdução e acima da cabeça (secar o cabelo, por exemplo), ou com uma pressão inferior (carregar pesos, por exemplo). Os sintomas associados à compressão vascular são descritos como pouco freqüentes, resultando a compressão arterial em fenômeno de Raynaud ou claudicação vascular e a compressão venosa em trombose ${ }^{(5)}$.

O envolvimento vascular na SDT pode ser determinado pela observação clínica. No entanto, a alteração na amplitude do pulso com a abdução do membro superior pode existir em 15\% de pessoas normais, não sendo um indicador fiável da SDT ${ }^{(6)}$. Em alguns casos, a angiografia pode estar indicada, particularmente quando se ausculta um sopro vascular supra ou infraclavicular, em caso de ausência de pulso radial ou de presença de uma massa paraclavicular pulsátil ${ }^{(5)}$.

\section{AVALIAÇÃO CLÍNICA}

São descritas quatro manobras provocadoras para o diagnóstico da SDT que, quando são positivas, reproduzem os sintomas nervosos e provocam diminuição da amplitude do pulso radial: 1) Teste de Adson: o doente vira a cabeça para o lado sintomático, estirando o pescoço e inspirando profundamente. 2) Teste de Halsted ou costoclavicular: o doente coloca os ombros em posição recuada e para baixo (postura militar), diminuindo o espaço costoclavicular. 3) Teste de Roos: elevação do membro superior a $90^{\circ}$ de abdução do ombro com rotação externa e flexão do cotovelo durante 3 minutos, associada a movimentos rápidos de abrir e fechar a mão. 4) Teste de Wright: hiperabdução do ombro a $180^{\circ}$ com flexão do cotovelo, durante 1 minuto. 
Nenhum desses testes foi aceito como gold standard para a SDT e como testes de insuficiência vascular tiveram muitos resultados falsos-negativos e falsos-positivos ${ }^{(5)}$.

\section{AVALIAÇÃO RADIOGRÁFICA}

As radiografias do tórax e da coluna cervical podem ser úteis para revelar alterações ósseas: costela cervical e grande apófise transversa de C7 são as classicamente descritas como associadas à SDT. A presença de uma costela cervical é descrita com uma prevalência de $1 \%$ na população geral e de $6 \%$ a $11 \%$ em doentes com SDT. A tomografia axial computorizada (TAC) pode ser útil no diagnóstico de SDT, particularmente nos pacientes em que as radiografias são normais, quer estabelecendo o diagnóstico de SDT, quer identificando outras causas para os sintomas ${ }^{(5)}$. A ressonância magnética tem-se mostrado útil, revelando bandas fibrosas e desvio do plexo braquial em doentes sem costela cervical ${ }^{(7)}$.

\section{AVALIAÇÃO ELETRODIAGNÓSTICA}

O estudo da velocidade da condução nervosa é mais útil na identificação de compressões nervosas distais, como a síndrome do canal cárpico ou do canal de Guyon, do que na confirmação do diagnóstico de SDT. Recentemente, os potenciais evocados somatosensitivos (PESS) foram apontados como medida mais sensível no diagnóstico. Estimulando a extremidade distal e registrando a resposta na fossa supraclavicular e na medula cervical, teoricamente os PESS podem refletir a alteração nervosa em nível do plexo braquial proximal ${ }^{(5)}$. Machleder et al. avaliaram os PESS em 80 doentes com SDT, tendo obtido $74 \%$ de alterações ${ }^{(8)}$. Pode ser difícil avaliar os doentes com SDT em uma postura neutra, pelo que recentemente se começou a aplicar os testes nas posições forçadas descritas. Machleder et al. e Borg et al. referem a PESS mais alterados quando os testes eram efetuados nestas posições ${ }^{(8,9)}$. Komanetsky et al., estudando os PESS em 21 doentes com SDT e em 23 controles, em posição relaxada e forçada, não consideraram esse teste útil para o diagnóstico ${ }^{(10)}$.

\section{AVALIAÇÃO ANGIOGRÁFICA}

A angiografia é um dos métodos de imagem que permitem identificar uma compressão arterial em doentes com diagnóstico clínico de SDT, ilustrando o local e tipo de estreitamento e a sua variação dinâmica com a mudança de posição do membro superior ${ }^{(11)}$. Os avanços tecnológicos e o surgimento da angiorressonância vieram trazer vantagem sobre a angiografia convencional, permitindo caracterizar, não só a anatomia arterial, como a dos tecidos envolventes e responsáveis pela compressão. A grande vantagem dessas técnicas seria a de evitar a realização de um exame mais invasivo, fornecendo como ele uma informação precisa sobre a anatomia vascular, mas também das estruturas ósseas, tendinosas e musculares envolventes ${ }^{(12-14)}$.

\section{RELATO DE CASO}

Adolescente de 16 anos, cor branca, estudante, observada em consulta de reumatologia com omalgia direita com 3 meses de evolução, acompanhada de disestesias do antebraço e mão homolaterais, que eram desencadeadas por movimentos de abdução do ombro. Referia há 7 meses trabalhar diariamente, durante cerca de 2 a 3 horas, com um computador. Na observação, a manobra de hiperabdução do membro superior desencadeava os sintomas e um enfraquecimento do pulso radial direito. Não se auscultavam sopros vasculares e, com o membro superior em posição neutra, os pulsos radiais eram amplos e simétricos. A pressão arterial em posição neutra do membro superior era de 128/67 mmHg à direita e 111/75 mmHg à esquerda. Em hiperabdução, a pressão arterial era de $75 / 49 \mathrm{mmHg}$ à direita e de 92/74 à esquerda.

Foi diagnosticada síndrome do desfiladeiro torácico à direita e realizada tomografia axial computorizada (TAC) na região torácica, que não revelou massas infra-claviculares nem axilares à direita. As análises feitas não revelaram alterações, nomeadamente dos parâmetros inflamatórios (velocidade de sedimentação e proteína C reactiva).

$\mathrm{O}$ ecodoppler do membro superior revelou uma onda trifásica na artéria subclávia, foi realizada uma angiografia das artérias subclávia e axilar direitas.

A angiografia do membro superior direito mostrou que as artérias subclávia e axilar tinham calibres normais, não se identificando alteração dos seus ramos colaterais. Com o braço em abdução havia uma discreta moldagem do contorno superior da subclávia distal, na transição com a artéria axilar, que era condicionada pela costela, mas sem alteração do calibre (Figura 1). Com o braço em abdução máxima, observava-se redução do contraste no interior da artéria axilar em uma extensão relativamente longa, sensivelmente ao nível do colo da omoplata. Essa alteração, embora sem ocorrer com irregularidade ou aparente redução do calibre da artéria, estaria eventualmente relacionada com um efeito de compressão no sentido ântero-posterior, que, no entanto, não se conseguia observar, pela limitação das incidências (Figura 2). 
O diagnóstico etiológico considerado mais provável para a SDT nesta paciente foi a hipotonia da musculatura elevadora do ombro. A orientação terapêutica consistiu em realização de fisioterapia, tendo a paciente sido ensinada a executar estiramentos dos membros superiores e exercícios de fortalecimento dos músculos elevadores dos ombros. Seis meses depois do início desse tratamento, houve grande melhoria dos sintomas.

\section{DISCUSSÃO}

O diagnóstico diferencial de uma SDT, principalmente por tratar-se de uma mulher jovem, inclui necessariamente a arterite de Takayasu ou "doença sem pulsos". Essa paciente não apresentava claudicação intermitente dos membros superiores e não tinha sintomas constitucionais. Os pulsos radiais eram amplos e simétricos, e não se auscultavam
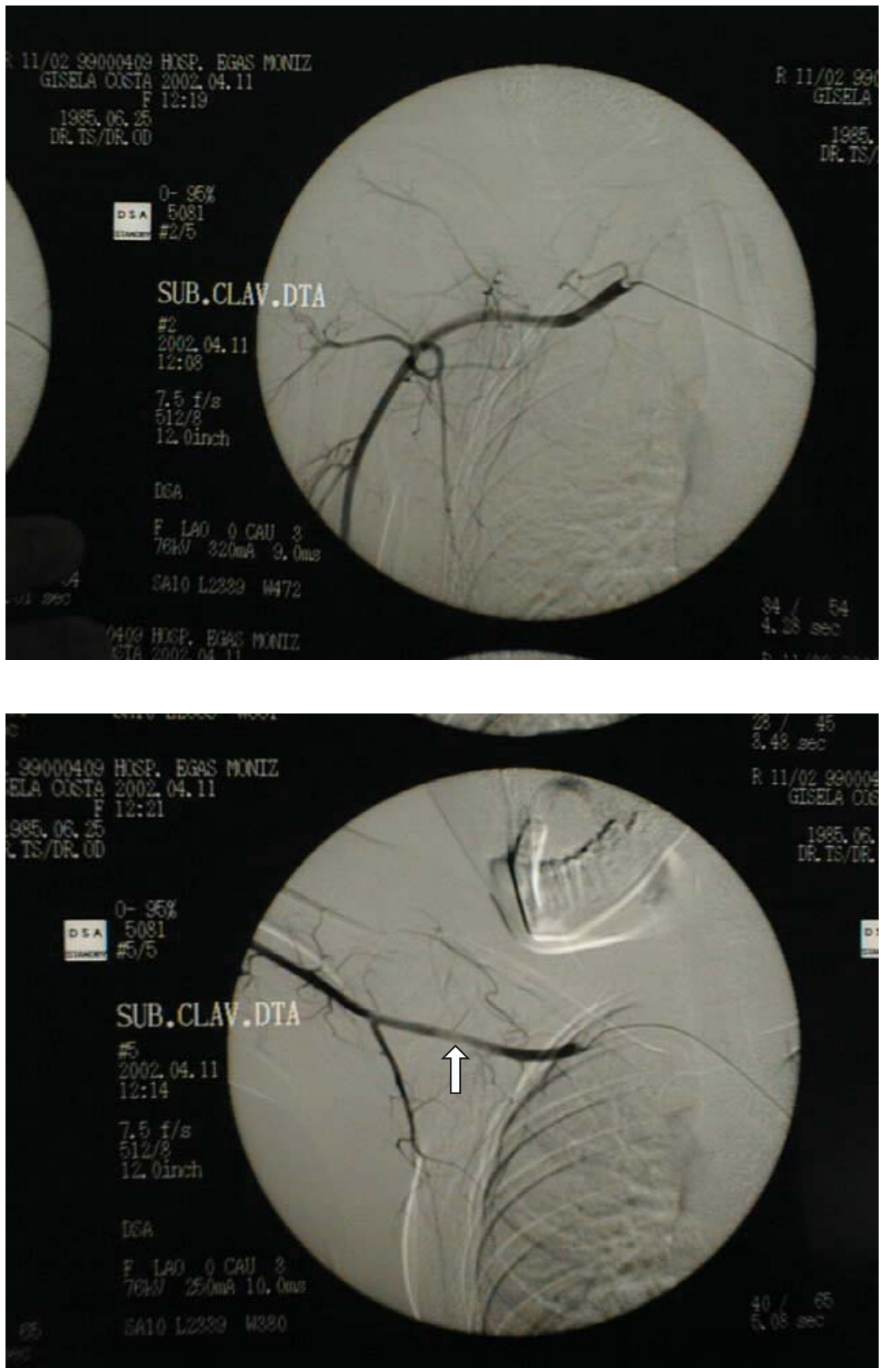

Figura 1 - Angiografia das artérias subclávia e axilar direitas com o membro superior em abdução.
Figura 2 - Angiografia das artérias subclávia e axilar direitas com o membro superior em abdução. 
sopros vasculares, pelo que este diagnóstico era menos provável. No entanto, a alteração arterial sugerida pelo ecodoppler levou à realização de uma angiografia, exame mais invasivo que permitiu confirmar a existência de uma compressão arterial com o membro superior em abdução, na ausência de estenoses e de aneurismas.

O doppler em cores é um exame de imagem útil no diagnóstico da compressão vascular, mostrando alterações significativas, ou seja, aumento da velocidade do fluxo, pré-oclusão e oclusão da artéria subclávia, com o braço em abdução. Por ser um exame não-invasivo, é uma alternativa à angiografia quando esta está contra-indicada ${ }^{(15)}$.

Em um estudo no qual foi utilizada a tomografia computadorizada (TC) helicoidal, esta mostrou existir um estreitamento significativo do espaço costo-clavicular após manobras posturais, com deslocamento da artéria subclávia ou axilar, em mais de metade dos doentes com diagnóstico de $\mathrm{SDT}^{(16)}$. Esse exame de imagem foi também utilizado em um grupo de atletas com sintomas de compressão arterial em nível do SDT, a qual se relaciona, neste grupo de pacientes, com uma hipertrofia de tecidos moles e sobreutilização, tendo localizado com precisão as lesões arteriais e os causadores da compressão $\mathrm{O}^{(17)}$.

Em um outro estudo realizado por autores portugueses, em 24 pacientes com diagnóstico clínico de SDT, a

\section{REFERÊNCIAS}

1. Wilbourn A J: Thoracic Outlet Syndromes. Neurologic Clinics 17(3): 477-97, 1999.

2. Peet PM, Henriksen MD, Anderson TP: Thoracic Outlet Syndrome: Evaluation of a Therapeutic Exercise Program. Mayo Clin Proc 31: 281-7, 1956.

3. Novak CB, Mackinnon SE: Thoracic Outlet Syndrome. Orthop Clin North Am 27(4):747-62, 1996.

4. Henriques M, Fernandes A, Ferreira AC: Síndrome VértebroTorácica. Acta Med Port 8:169-71, 1995.

5. Mackinnon SE, Novak CB: Evaluation of the Patient With Thoracic Outlet Syndrome. Seminars in Thoracic and Cardiovascular Surgery 8(2):190-200, 1996.

6. Dawson DM: Entrapment Neuropthies of the Upper Extremities. N Engl J Med 329(27):2013-8, 1993.

7. Urschel JD, Hameed SM, Grewal RP: Neurogenic Thoracic Outlet Syndromes. Postgrad Med J 70:785-9, 1994.

8. Machleder HI, Moll F, Nuwer M, et al: Somatosensory Evoked Potentials in the Assessment of Thoracic Outlet Syndrome. J Vasc Surg 6:177-84, 1987.

9. Borg K, Persson HE, Lindblom U: Thoracic Outlet Syndome: Diagnostic Value of Sensibility Testing, Vibratory Thresholds and Somatosensory Evoked Potentials at Rest and During Perturbation With Abduction and External Rotation of the Arm. Proceedings from the Fifth World Congress on Pain, Amsterdam, Elsevier:144$50,1988$. angiografia confirmou o diagnóstico em 7 pacientes e revelou outros diagnósticos em 3. Em 14 pacientes o exame era normal ${ }^{(11)}$. Outros autores, em um artigo de 1975, advogavam que a arteriografia posicional devia ser reservada para os doentes com suspeita de lesão arterial como aneurisma, obstrução ou fenômenos embólicos, visto esta técnica não ter utilidade comprovada nas compressões da $\mathrm{SDT}^{(18)}$.

Mais recentemente, quer a angiorressonância magnética tridimensional ${ }^{(12,13)}$ quer a angiorressonância ultra-rápida de alta resolução ${ }^{(14)}$ mostraram resultados comparáveis aos da angiografia convencional, podendo evitar a realização desta e tendo possibilitado a identificação precisa da estrutura causadora da compressão vascular em pacientes com diagnóstico clínico de SDT, como, por exemplo, um osteófito clavicular que causava compressão arterial ${ }^{(13)}$ e demonstrado o efeito da hiperabdução do membro superior na reprodução dos sintomas ${ }^{(12,14)}$.

Não sendo esses últimos exames de imagem facilmente disponíveis, os autores sugerem que a angiografia convencional das artérias subclávia e axilar deva ser realizada nos doentes com diagnóstico clínico sugestivo de SDT com envolvimento vascular, para localizar a compressão arterial e excluir o diagnóstico de arterite de Takayasu.

10. Komanetsky RM, Novak CB, Mackinnon SE, et al: Somatosensory Evoked Potentials Fail to Daignose Thoracic Outlet Syndrome. J Hand Surg 19A: 873-83, 1994.

11. Bogalho L, Seixas I, Martins JM, Pisco JM: Angiography in Thoracic Outlet Syndrome. Acta Med Port 11(1):33-6, 1998.

12. Dymarkowski S, Bosmans H, Marchal G, Bogaert J: ThreeDimensional MR Angiography in the Evaluation of Thoracic Outlet Syndrome. AJR Am J Roentgenol 173(4):1005-8, 1999.

13. Tobalina I, Alvaro-Gonzalez LC, Garcia-Andrade L: Arterial Thorcic Outlet Syndrome and Diagnostic Angioresonance. Rev Neurol 24(136):1541-2, 1996.

14. Hagspiel KD, Spinosa DJ, Angle JF, Matsumoto AH: Diagnosis of Vascular Compression at the Thoracic Outlet Using GadoliniumEnhanced High-Resolution Ultrafast MR Angiography in Abduction and Adduction. Cardiovasc Intervent Radiol 23(2):152-4, 2000.

15. Wadhwani R, Chaubal N, Sukthankar R, Shroff M, Agarwala S: Color Doppler and Duplex Sonography in 5 Patients With Thoracic Outlet Syndrome. J Ultrasound Med 20(7):795-801, 2001.

16. Remy-Jardin M, Remy J, Masson P, et al: Helical CT Angiography of Thoracic Outlet Syndrome: Functional Anatomy. AJR Am J Roentgenol 174(6):1667-74, 2000.

17. Yao JS: Upper Extremity Ischemia in Athletes. Semin Vasc Surg 11(2):96-105, 1998.

18. Sadler TR, Rainer WG, Twombley G: Thoracic Outlet Compression. Application of Positional Arteriographic and Nerve Conduction Studies. Am J Surg 130(6):704-5, 1975. 